Factores de motivación de logro: el compromiso y entrega en el aprendizaje, la competencia motriz percibida, la ansiedad ante el error y situaciones de estrés en estudiantes de cuarto, quinto y sexto nivel escolar durante la clase de educación física

Márquez-Barquero, Magally; Azofeifa-Mora, Christian; Rodríguez-Méndez, Diego

Factores de motivación de logro: el compromiso y entrega en el aprendizaje, la competencia motriz percibida, la ansiedad ante el error y situaciones de estrés en estudiantes de cuarto, quinto y sexto nivel escolar durante la clase de educación física

Revista Educación, vol. 43, núm. 1, 2019

Universidad de Costa Rica, Costa Rica

Disponible en: http://www.redalyc.org/articulo.oa?id=44057415005

DOI: https://doi.org/10.15517/revedu.v43i1.33109

Esta obra está bajo una Licencia Creative Commons Atribución-NoComercial-SinDerivar 3.0 Internacional. 


\title{
Factores de motivación de logro: el compromiso y entrega en el aprendizaje, la competencia motriz percibida, la ansiedad ante el error y situaciones de estrés en estudiantes de cuarto, quinto y sexto nivel escolar durante la clase de educación física
}

Factors of motivating achievement: the commitment and dedication in learning, perceived motor competence, anxiety about the error and stress situations in fourth, fifth and sixth grade students during physical education class

\author{
Magally Márquez-Barquero \\ Universidad Nacional, Costa Rica \\ magally.marquez.barquero@una.cr \\ Christian Azofeifa-Mora \\ Universidad Nacional, Costa Rica \\ cristian.azofeifa.mora@una.cr \\ Diego Rodríguez-Méndez \\ Universidad Nacional, Costa Rica \\ diego.rodriguez.mendez@una.cr
}

DOI: https://doi.org/10.15517/revedu.v43i1.33109

Redalyc: http://www.redalyc.org/articulo.oa?id=44057415005

\author{
Recepción: 01 Julio 2018 \\ Aprobación: 24 Noviembre 2018
}

\section{Resumen:}

Objetivo: determinar los factores asociados a la motivación de logro (ML) compromiso y entrega en el aprendizaje (CEA), competencia motriz percibida (CMP) y ansiedad ante el error (AE) durante las clases de Educación Física en el estudiantado que cursa el II ciclo de la Educación General Básica. Metodología: Se aplicó el cuestionario AMPET a un total de 323 escolares. Se realizó el análisis descriptivo de promedios y desviaciones estándar, análisis ANOVA 2x3 (2 sexos y 3 niveles escolares), para grupos independientes y post hoc de Tukey. El nivel de significancia establecido fue $\mathrm{p}<.05$. Resultados: se encontraron diferencias en el factor de CEA según el nivel ( $\mathrm{p}=.000$ ). a menor grado académico mayor puntuación, el estudiantado de cuarto nivel refleja un mayor CEA que el de quinto y sexto. El factor CMP según el nivel, arrojó que a mayor nivel académico el puntaje disminuye $(\mathrm{p}=.000)$ y fue el estudiantado de cuarto nivel quien mostró una puntuación más alta en comparación con los otros niveles. El factor AE y las situaciones de estrés, mostró mayores puntuaciones en las mujeres ( $\mathrm{p}=.007)$ en relación a los hombres. Conclusión: Factores como CEA, CMP y AE, relacionadas a la ML en las clases de EF, juegan un papel motivacional, las bajas percepciones de la CMP observadas, son señal de desmotivación e insuficiente participación en las actividades de la clase amenazando con un menoscabo en la competencia motriz real y en el factor AE según el sexo, la mirada debe enfocarse hacia realizar refuerzos positivos para disminuir los niveles de estrés y ansiedad que se generan debido y durante la clase de EF, focalizando la atención hacia las mujeres, debido a que se observó que son ellas quienes presentaron los puntajes negativos.

Palabras Clave: Comportamiento, formación deportiva, niñez, AMPET.

\section{Abstract:}

Design: longitudinal, observational, prospective descriptive study. Objective: to determine the factors associated to the motivation of achievement (ML) commitment and delivery in learning (CEA), perceived motor competence (CMP) and anxiety before the error (AE) during the Physical Education classes in the students that attends the II cycle of Basic General Education. Methodology: The AMPET questionnaire was applied to a total of 323 schoolchildren. The descriptive analysis of averages and standard deviations, ANOVA 2x3 parametric analysis (2 sexes and 3 school levels), for independent and post hoc groups of Tukey was applied. The level of significance established was $\mathrm{p}<.05$. Results: differences were found in the CEA factor according to the level $(\mathrm{p}=.000)$. The lower the academic grade, the higher the score; the fourth-level students reflects a higher CEA than the fifth and sixth grades. The CMP factor according to the level, showed that the higher the academic level the score decreases $(p=.000)$, and it was the fourth level student who showed a higher score compared to the other levels. The AE factor and stress situations showed higher scores in women $(\mathrm{p}=.007)$ in relation to men. Conclusion: Factors such as CEA, CMP and AE, related to the ML in the PE classes, play a motivational role. The low perceptions of the CMP observed, are a sign of demotivation and insufficient participation in the activities of the class, threaten with a diminution in real motor competition and in the AE factor according 
to sex. The analysis must be focused on positive reinforcement to reduce the levels of stress and anxiety that are generated due to and during PE class, focusing attention on women, since it was observed that they presented the negative scores.

KEYWORDS: Behavior, sports training, childhood, AMPET.

\section{INTRODUCCIÓN}

Se reconoce que la Educación Física (EF) juega un papel potencialmente importante en la mejora de la salud pública mediante las actitudes relacionadas hacia el ejercicio y la promoción de programas de acondicionamiento físico concernientes con la salud (Ntoumanis, 2001), la EF permite un desarrollo de la personalidad, de valores y de una diversidad de patrones y movimientos de los educandos, vinculándolo a un enfoque para la vida (Ministerio de Educación Pública, 2013; Gutiérrez, 2004).

La motivación es el conjunto de factores internos y externos que motivan y determinan las acciones de una persona (Real Academia Española, 2018), ha sido estudiada desde diferentes campos, donde la personalidad posee un papel preponderante y una relevancia de integrar diversas categorías psicológicas como la autovaloración, la necesidad, los motivos de práctica, la afectividad que en ella implica y la actitud ante la tarea (Becerra-González y Reidl, 2015; Durán-Aponte y Elvira-Valdés, 2015; Rosales, 2011).

La perspectiva humanista de la motivación expresa la capacidad que poseen las personas para crecer, a partir de lo anterior subyacen diversas teorías como la de las necesidades de Maslow, la teoría de existencia, relación y crecimiento de Alderfer, (Naranjo-Pereira, 2009). Sobre esta línea humanista de la motivación, suscitada en el ámbito escolar, la teoría de las necesidades de McClelan, donde las divide en tres ámbitos: la necesidad de afiliación, la necesidad de poder y la necesidad de la metas de logro para la resolución de conflictos (Huertas, 1997; Naranjo-Pereira, 2009).

El segundo enunciado es sobre la teoría de la motivación de metas de logro de Atkinson, la cual expresa que el estudiante se encuentra motivado buscando el éxito y evitando el fracaso (Huertas, 1997; NaranjoPereira, 2009 y Ruiz et ál., 2004) .

Bajo estos lineamientos básicos, la motivación de logro (ML) en el aprendizaje del estudiantado en EF se basa en los enunciados de McClelan y más precisamente en los enunciados de Atkinson sobre la motivación de las metas de logro, donde el estudiantado basa su motivación a la conducta de esforzarse buscando el éxito, al esfuerzo de lograr mejores rendimientos, dentro de un estándar ideal de logro y por consiguiente disminuir la tendencia al fracaso en dicha tarea, es la motivación para la superación de retos, obstáculos o problemas (Astorga y Ojeda, 2009; Romero, 1981).

Precisamente la evaluación realizada por el Test de Motivación de logro para el Aprendizaje en Educación Física o AMPET (Achievement Motivation in Physical Education Test, por sus siglas en inglés), se basa predominantemente en la teoría de Atkinson desde su versión original y específicamente utilizado en las clases de educación física (Coterón, Álvarez, Pérez-Tejero, \& Sampedro, 2013; Ruiz-Pérez, Moreno-Murcia, Ramón-Otero, \& Alias-García, 2015; Ruiz-Pérez, Ruiz-Amengual, \& Linaza-Iglesias, 2016; Ruiz, Graupera, Contreras, \& Nishida, 2004; Sánchez Alcaraz \& Gómez Mármol, 2016).

Las clases de EF apoyadas en prácticas asociadas a la ML, se ven influenciadas por una serie de factores inherentes dentro de los cuales destacan el CEA, que se define como aquel nivel donde la persona participa de forma activa para adquirir nuevo conocimiento y se pone de manifiesto los niveles de intensidad y emotividad, consecuencia de empezar nuevos aprendizajes (Turabik \& Baskan, 2015; Yamila \& Donolo, 2014).

Otro factor importante es la competencia motriz percibida (CMP) que en su concepto se relaciona con la capacidad con la cual pueden actuar con eficiencia para desarrollar cada una de las tareas encomendadas en las clases de EF (López, Camerino, \& Castañer, 2015) y por último, está el factor ansiedad ante el error y los factores de estrés (AE), conceptualizado como el temor generado por la posibilidad de fracasar durante las diferentes tareas que se desarrollan en las clases de EF (Coterón, Álvarez, Pérez-Tejero, \& Sampedro, 2013; Lonsdale et ál., 2015). 
Dado a lo anterior, en la motivación de logro según Atkinson expone que la recompensa adquirida por la motivación de logro es el logro en sí mismo, y dentro del campo de la EF el estudiantado más motivado hacia el logro, presentará mejores rendimientos en el aprendizaje de las habilidades motrices, en comparación con los estudiantes que expresan una motivación para evitar el fracaso (Nishida y Inomata, 1981; Romero, 1981).

Para Coterón, Bernabé y Franco (2010); Halvari, Skjesol, y Bagøien (2011) señalan que, en los últimos años, se han realizado investigaciones para estudiar la motivación del estudiantado hacia el aprendizaje durante las lecciones de EF y que se ha desarrollado bajo modelos cognitivo-sociales.

Los estudios en la motivación y la EF se han enfocado de múltiples maneras en distintos países como Japón, Estados Unidos, Canadá e Inglaterra (Ruiz, Graupera, Contreras, y Nishida, 2004), por ejemplo, se han realizado estudios enfocados en el clima motivacional (Campos, Vicedo, Villora, y Madrona, 2010; Coterón, Franco, Pérez-Tejero y Sampedro, 2013; Halvari et ál., 2011; Wallhead y Ntoumanis, 2004); en la autodeterminación (Moreno, González-Cutre, Martín-Albo y Cervelló, 2010; Ntoumanis, 2001); en la ansiedad (Quevedo-Blasco, Quevedo-Blasco y Bermúdez, 2009) y en las diferencias de sexo (Coterón et ál., 2010; Gómez, Gámez y Martínez, 2011; Ntoumanis, 2001).

En el ámbito escolar, los estudios señalan que en cuanto a la motivación hacia la clase de EF, el clima motivacional orientado a la tarea, la CMP y la AE, presentan una tendencia a mejorar (Coterón et ál., 2013; Halvari et ál., 2011; Wallhead y Ntoumanis, 2004).

Las investigaciones orientadas hacia las diferencias entre sexos, Ntoumanis (2001) mostró que las mujeres están más motivadas que los hombres pero de manera intrínseca según la afiliación; por el contrario, en el estudio de Gómez et ál., (2011) demostró que en la clase de EF las mujeres se encuentran más desmotivadas que sus pares y en el estudio de Coterón et ál. (2010) confirmaron que las mujeres presentaron menores resultados en el CEA y la CMP, así como mayores puntuaciones en la AE que los hombres.

En Costa Rica las investigaciones en EF se han centrado en la importancia de la EF en el currículo educativo y su impacto positivo en la educación (Vargas-Pérez y Orozco-Vargas, 2003); en el rol promotor de valores en la etapa escolar (Flores-Moreno y Zamora-Salas, 2009; Márquez-Barquero, 2015) y en el campo de los motivadores de los profesionales en EF (Orozco-Vargas, 2010, 2013; Vargas-Pérez y Orozco-Vargas, 2003).

A nivel escolar, se ha trabajado en la detección de factores socioafectivos en programas de intervención motriz (Herrera-González, Delgado-Tenorio, Fonseca-Schmidt y Vargas-Ramírez, 2012), en el perfil motriz y psicológico en cuanto a autoestima y auto concepto (Herrera-González, Morera-Castro, Araya-Vargas, Fonseca-Schmidt y Vargas-Ramírez, 2017); en el sedentarismo (Álvarez-Bogantes, 2006) y en la satisfacción del estudiantado con la clase de EF (Orozco-Vargas, 2013; Ruiz-Juan y Baena-Extremera, 2015).

Debido a la evidencia científica en el ámbito escolar costarricense y a la importancia que reviste el tema de la motivación de logro para incentivar estilos de vida saludables asociados al desempeño motriz desde edades tempranas, resulta necesario indagar los factores que inciden en la motivación de logro en el estudiantado, con el fin de aportar conocimiento para orientar los procesos metodológicos de las clases de EF; por lo anterior, el propósito de la investigación fue determinar los factores asociados a la ML en el estudiantado de II ciclo según el sexo y nivel escolar.

\section{Metodología}

\section{Diseño}

La investigación se desarrolló como estudio descriptivo longitudinal, observacional, prospectivo, las variables tratadas no se vieron manipuladas por la aplicación de un tratamiento determinado y el fin primordial fue describir el comportamiento de las variables por medio de la recolección de datos con un instrumento en el grupo de participantes. 


\section{Participantes}

La selección de la muestra se realizó con el método no probabilístico por conveniencia, lo cual significa que se trabajó con ellos por su disponibilidad voluntaria. Se reclutó a trescientos veintitrés participantes, quienes cursaban cuarto, quinto y sexto año de primaria, todos provenientes de seis centros educativos públicos ubicados en el cantón Central de la Provincia de Heredia. Los criterios de elegibilidad fueron: la entrega de la autorización formal del asentimiento y el consentimiento informado emitido por la persona encargada legal y que no presentaran ningún tipo de discapacidad cognitiva, ni motora. En la tabla 1 se resumen las características de los participantes.

TABLA 1

Tabla 1 Distribución de la cantidad de estudiantes de II ciclo en función de la edad según el sexo, nivel educativo

\begin{tabular}{|c|c|c|c|c|c|c|}
\hline & \multicolumn{2}{|c|}{ Hombres } & & \multicolumn{2}{|c|}{ Mujeres } & \\
\hline & $n$ & Edad & & $\mathrm{n}$ & Edad & \\
\hline Nivel & & $\square$ & $\mathrm{DE}$ & & $\square$ & $\mathrm{DE}$ \\
\hline Cuarto & 52 & 10 & 0.57 & 56 & 9.89 & 0.60 \\
\hline Quinto & 58 & 10 & 0.52 & 50 & 10 & 0.55 \\
\hline Sexto & 58 & 12 & 0.55 & 49 & 11 & 0.56 \\
\hline
\end{tabular}

En la tabla 1 se muestran las características más importantes de la población de estudiantes en primaria relacionadas con la cantidad y edades promedio según el sexo y nivel educativo. Cabe mencionar, que dicha población debe realizar 80 minutos de actividades relacionadas con las temáticas de la educación física por semana según lo establecen las políticas curriculares vigentes.

\section{Instrumentos}

Se utilizó el Test AMPET de motivación de logro para el aprendizaje en Educación Física (Achievement Motivation in Physical Education Test); el cual analiza los factores compromiso y entrega en el aprendizaje; competencia motriz percibida y ansiedad ante el error en las situaciones de estrés, las consistencias internas (alpha de Cronbach) de los tres factores fueron de .86, .82 y .87 respectivamente (Ruiz et ál., 2004); ha sido aplicado a poblaciones en edad escolar (López, Álvarez, Pérez-Tejero \& Molinuevo, 2013 ; Ruiz-Pérez, Moreno-Murcia, Ramón-Otero \& Alias-García, 2015 ; Ruíz Pérez, 2014 y Sánchez Alcaraz, Bernardino Javier; Gómez Mármol, 2016) ; cuenta con 37 ítems agrupados en tres dimensiones: CEA ("Me concentro en lo que tengo que practicar durante la clase de educación física"); CMP ("Para las clases de educación física, pienso que poseo mejores capacidades que otros compañeros y compañeras") y AE ( "En la mayoría de las clases de educación física me pongo nervioso/a cuando tengo que realizar los ejercicios delante de mis compañeros/as del grupo y esto hace que no dé lo mejor de mí”). Las respuestas deben darse en una escala tipo Likert con cinco alternativas, desde (1) totalmente en desacuerdo, hasta (5) totalmente de acuerdo.

\section{Procedimiento}

Por las características de la población se estableció una logística de trabajo desarrollada en dos fases. 


\section{Fase I}

Se visitó los centros educativos para coordinar con la administración los permisos de acceso y reclutamiento de los participantes y hacer la entrega de los consentimientos y los asentimientos informados. Se acordó el día y la fecha de la aplicación del instrumento con el profesional de educación física responsable del grupo de participantes.

\section{Fase II}

Para la aplicación del cuestionario se contó con siete colaboradores quienes reunieron a los participantes en el aula y una vez ahí se le entregó un documento por persona, se procedió a leer las instrucciones y a evacuar dudas, posteriormente cada participante contestó por escrito a las preguntas de los enunciados del Test de AMPET.

\section{Análisis estadístico}

Se realizó el análisis descriptivo promedios y desviaciones estándar, así como análisis paramétrico de ANOVA una vía $2 \times 3$ ( 2 sexos y 3 niveles escolares), para grupos independientes y post hoc para conocer qué relaciones específicas se diferencian entre sí en cada factor y qué relaciones específicas hay entre los grupos. El nivel de significancia establecido fue $\mathrm{p}<.05$. Se empleó el paquete estadístico IBM SPSS Statistics $@ 24$ versión para Windows.

\section{Resultados}

En la tabla 2 se observan los resultados (promedios y desviaciones estándar) de los tres factores, según el nivel y el sexo.

TABLA 2

Tabla 2 Promedio y desviación estándar de las Dimensiones del cuestionario AMPET según el sexo y nivel educativo

\begin{tabular}{llllll}
\hline & Sexo & \multicolumn{4}{l}{ Nivel Educativo } \\
\hline Factor & Hombre & Mujer & Cuarto & Quinto & Sexto \\
\hline $\begin{array}{l}\text { Compromiso } \\
\text { y entrega } \\
\text { en el }\end{array}$ & $4.14 \pm .045$ & $4.09 \pm .047$ & $4.34 \pm .057$ & $4.09 \pm .057$ & $3.92 \pm .057$ \\
aprendizaje & & & & & \\
\hline $\begin{array}{l}\text { Competencia } \\
\text { motriz }\end{array}$ & $3.08 \pm .067$ & $2.99 \pm .070$ & $3.34 \pm .083$ & $3.02 \pm .083$ & $2.75 \pm .084$ \\
percibida & & & & & \\
$\begin{array}{l}\text { Ansiedad } \\
\text { ante el error }\end{array}$ & & & & & \\
$\begin{array}{l}\text { y las } \\
\text { Situaciones } \\
\text { de estrés }\end{array}$ & $2.18 \pm .072$ & $2.46 \pm .075$ & $2.23 \pm .090$ & $2.40 \pm .090$ & $2.34 \pm .090$ \\
\hline
\end{tabular}

Elaboración propia 
TABLA 3

Tabla 3 Análisis de las Dimensiones del cuestionario AMPET en función del sexo, nivel educativo

\begin{tabular}{lllllll}
\hline & Sexo & \multicolumn{3}{c}{$\begin{array}{l}\text { Nivel de } \\
\text { Primaria }\end{array}$} & \multicolumn{3}{c}{$\begin{array}{l}\text { Sexo x Nivel } \\
\text { de Primaria }\end{array}$} \\
\hline Factor & F & P & F & sig & F & sig \\
\hline $\begin{array}{l}\text { Compromiso y } \\
\text { entrega en el } \\
\text { aprendizaje }\end{array}$ & .557 & .456 & 13.903 & $.000^{*}$ & .761 & .468 \\
\hline $\begin{array}{l}\text { Competencia } \\
\text { Motriz Percibida }\end{array}$ & .783 & .377 & 12.353 & $.000^{*}$ & .996 & .371 \\
\hline $\begin{array}{l}\text { Ansiedad ante el } \\
\text { error y las } \\
\text { Situaciones de } \\
\text { estrés }\end{array}$ & 7.38 & $.007^{*}$ & .934 & .394 & 3.72 & .025 \\
\hline
\end{tabular}

${ }^{*} \mathrm{p}<.05$

Elaboración propia

Se encontraron diferencias significativas en el factor de compromiso y entrega en el aprendizaje según el nivel de primaria $(\mathrm{p}=.000)$. El post-hoc de Bonferroni, mostró que a menor grado académico mayor es el puntaje en este factor; es decir, el estudiantado de cuarto nivel refleja un mayor compromiso y entrega para el aprendizaje en lo relacionado a la adquisición de nuevo conocimiento en las clases de EF, con respecto al estudiantado de quinto y sexto nivel. Para el factor de competencia motriz percibida según el nivel de primaria, el post-hoc de Bonferroni $(\mathrm{p}=.000)$ arrojó que a mayor nivel académico el puntaje disminuye y fue el estudiantado de cuarto nivel quien mostró una puntuación más alta en comparación con quinto y sexto nivel, denotando una mejor percepción sobre sus habilidades motrices para encarar las tareas propias de las clases de EF. Con respecto al factor ansiedad ante el error y las situaciones de estrés, las mujeres obtuvieron un puntaje más elevado con respecto a los hombres, mostrando una mayor percepción de ansiedad ante el error en las actividades propias de EF y que estas pueden desencadenar en estadios de estrés ( $\mathrm{p}=.007$ ) (tabla 2 y 3 ).

\section{Discusión}

Comprender los factores que motivan al estudiantado a su participación en las clases de EF, resulta determinante tanto para los profesionales del área; así como, para las autoridades respectiva para la toma de decisiones relacionadas al desarrollo de estrategias educativas presentes y futuras (Lonsdale et ál., 2015; RuizPérez et ál., 2015). Por lo anterior, el presente estudio se focalizó en determinar cuáles factores asociados a la motivación de logro en los estudiantes de II ciclo de la Educación General Básica durante las clases de EF, poseen mayores o menores atribuciones según el nivel educativo y el sexo.

$\mathrm{Al}$ ser analizado el factor de compromiso y entrega en el aprendizaje, se muestran resultados que reflejan una disminución en la percepción del estudiantado conforme se incrementa el nivel educativo, fenómeno que concuerda con otros estudios previos en poblaciones similares (Carrasco, Parra y Pérez, 2015), lo cual indica una menor participación de forma activa y consiente del estudiante, para adquirir nuevo conocimiento, manifestándose menores niveles de intensidad y emotividad para emprender actividades que le generen un nuevo aprendizaje (Turabik y Baskan, 2015; Yamila y Donolo, 2014). Tal comportamiento hacia un menor compromiso de adquirir nuevo aprendizaje, puede asociarse a modificaciones propias del ritmo de madurez evolutiva del ser humano, en donde conforme aumenta la edad, la motivación paulatinamente se sustenta en mayor medida a factores relacionados con motivaciones de ego (centrada en el reto y la constante comparación con los demás), pero no tanto en la motivación de logro la cual se enfoca prioritariamente en la consecución de objetivos planteados (López, Camerino, y Castañer, 2015; Sánchez-Alcaraz et ál., 2016). 
En lo referente al factor de competencia motriz percibida, se observa una disminución en sus puntuaciones conforme el nivel educativo aumenta, mismos resultados que concuerdan con investigaciones previas; tale resultados, reflejan una menor percepción en las habilidades motrices que cada estudiante posee para encarar con eficiencia, el desarrollo de cada una de las tareas asignadas en las clases de EF. Ello puede ser producto, de una menor práctica de ejercicio físico o deporte tanto durante las clases de EF como en otros espacios de esparcimiento, lo cual trae como consecuencia, una desaceleración en la estimulación motora y confianza generada por una menor participación; de igual forma, una mayor inclinación hacia una motivación asociada a la competitividad y la comparación con los demás, puede provocar una menor conciencia sobre la mejorar de la ejecución motriz correcta (Hellín, Moreno y Rodríguez, 2006; Carrasco et ál., 2015; López et ál., 2015)

Para el factor de ansiedad ante el error y las situaciones de estrés, las mayores puntuaciones en las mujeres con respecto a los hombres durante el desarrollo de las clases de EF, concuerdan con otros estudios realizados previamente y nos muestran una mayor tendencia en ellas, a percibir la posibilidad a fracasar en las actividades propias de las clases de EF, como algo relevante y estresante; lo anterior, puede ser el reflejo de una respuesta sobre estereotipos sociales y culturales aún arraigados en la sociedad, en donde se asocia a las mujeres con actividades más de elegancia o delicadeza y en menor medida con el ejercicio o deporte, lo cual resulta en juicios de valor, que son incompatibles con la naturaleza de actividades físico-deportivas, provocando así un déficit en el desarrollo motriz y que incide de forma directa, sobre la naturaleza y desarrollo de dichas clases; por otro lado, podría considerarse que las mujeres al tener menor participación, su competencia motriz esta menos estimulada y con ello la confianza para participar se ve mermada, lo cual puede recaer en estadios de estrés y desmotivación (Coterón et ál., 2013; López et ál., 2015; Sánchez-Alcaraz et ál., 2016; Lonsdale et ál., 2015).

\section{Conclusiones}

Los factores como el compromiso y la entrega; la competencia motriz percibida y la ansiedad ante el error y situaciones de estrés, se han asociado a la motivación de logro en las clases de EF, debido a que en esta busca generar un espacio para que la persona tenga la posibilidad de crear un entorno en el que pueda saciar sus deseos, emocionales, físicos y sociales, todas ellas como parte del desarrollo psicomotor.

La EF es una disciplina multifactorial que influye el desarrollo de la motivación, siendo así un aspecto psicológico que merece ser analizado desde diferentes perspectivas, para así entenderla y poder brindar herramientas para que el profesional en la materia, pueda conocer cómo se perciben y cómo se comparan los educandos con sus pares, qué es lo que les motiva y qué les limita para aprender y participar.

Bajas percepciones de la competencia motriz observadas, son señal de desmotivación, escaso compromiso a la tarea y la posibilidad de generar antipatía hacia la materia y como consecuencia insuficiente participación en las actividades de la clase que amenazan con un menoscabo en la competencia motriz.

Los especialistas en movimiento humano, son quienes están llamados a proponer estrategias de intervención efectivas para mejorar la motivación hacia la práctica deportiva y otras actividades afines y valerse de las condiciones positivas de CEA y CMP, observadas en cuarto nivel y tomarlo como el punto de partida para potenciar las destrezas motoras básicas, enfocar los contenidos del plan de estudios y atender las demandas de la población meta, con el propósito de generar un mayor bagaje en propuestas prácticas y apoyar el desarrollo motor que es el punto medular en esta área del comportamiento humano.

Además, quinto y sexto nivel presentaron una disminución paulatina del CEA, por lo tanto, se debe contemplar este hallazgo para hacer los ajustes y promover actividades donde se motive al estudiantado en los aprendizajes relacionados con la EF y como consecuencia intervenir la CMP.

En cuanto a las diferencias en el factor AE según el sexo, debe enfocarse a realizar refuerzos positivos para disminuir los niveles de estrés y ansiedad los cuales se generan durante la clase de EF, focalizando la atención hacia las mujeres, pues se observó que presentaron puntajes negativos y atribuidos muy probable a una baja 
CMP, como resultado de poco contacto o estímulo con las actividades físicas y deportivas, asociado a los roles que se asignan socialmente quienes se encuentran relacionados al sexo y que las aíslan de situaciones afines a la actividad física, las cuales marcan esa divergencia entre sexos y esto explica que las tareas motrices realizadas durante la clase de EF, fueron motivantes para una sola parte del estudiantado, por lo que se recomienda realizar una reflexión para una la clase más inclusiva según las características de ambos sexos.

A partir de los resultados obtenidos, se recomienda realizar estudios en el sector de la educación privada y en el sector de la educación pública en zona rural; también analizar el comportamiento en los factores relacionados a la motivación de logro y hacer diseños internacionales que contemplen la aleatorización y grupos o condiciones como controles, para conocer el efecto agudo y crónico de la sesión de EF basada en la competencia motriz, sobre los factores competencia motriz percibida y la ansiedad ante el error en la población de sexo femenino que fue en la cual se observaron resultados que merecen someterse a estudios más profundos.

Por otra parte, realizar investigación en primer ciclo de la educación general básica y a nivel longitudinal, esto con el fin de ofrecer al profesional en movimiento humano, información de los factores ML en esta población y por último, combinar posibles evaluaciones ML con diversas metodologías de trabajo para la búsqueda de un modelo acorde al nivel y el sexo del estudiantado.

\section{LIMITACIONES}

Se realizó el estudio descriptivo de los factores que afectan la motivación de logro y solo se analizó un sector de la educación pública y en zona urbana. La población meta, fue de difícil acceso, debido a que queda a criterio de la administración de cada centro educativo, permitir la ejecución de este tipo de investigaciones y en los lugares que sí aceptaron, fue difícil completar las diferentes fases, debido a la inestabilidad del cumplimiento del calendario escolar y a las diversas actividades extracurriculares que obstaculizan los procesos educativos.

Se agradece a todas las personas que colaboraron en la recolección de la información.

\section{REFERENCIAS}

Álvarez, C. (2006). Sedentarismo en niños costarricenses: sí hay solución. MHSALUD: Revista Ciencias del Movimiento Humano y Salud, 3(2). Retrieved from http://www.revistas.una.ac.cr/index.php/mhsalud/article/ view/334/10907

Astorga, L. y Ojeda, S. (2009). La motivación de logro y expectativas de vida en estudiantes de la licenciatura en psicología educativa de la U. P. N., Ajusco (Tesis de pregrado). Universidad Pedagógica Nacional, Perú.

Becerra, C., y Reidl, L. (2015). Motivación, autoeficacia, estilo atribucional y rendimiento escolar de estudiantes de bachillerato. Revista Electrónica de Investigación Educativa, 17(173), 79-93. Retrieved from http:// redie.uabc.mx/vol17no3/contenido-becerra-reidl.html

Campos, R. C., Vicedo, J. C. P., Villora, S. G., y Madrona, P. G. (2010). Educación física intercultural, motivación y prejuicio racial: una aproximación desde la teoría de las metas de logro. Cuadernos de Psicología Del Deporte, 10(2). Retrieved from http://revistas.um.es/cpd/article/view/111251/105601

Carrasco, M., Parra, D., y Pérez, C. (2015). La competencia motriz y su percepción en el alumnado de cuarto curso de Educación Secundaria y Bachillerato. Calidad de Vida y Salud, 8(2), 70-87. Retrieved from http:// revistacdvs.uflo.edu.ar/index.php/CdVUFLO/article/view/119/128

Coterón, J., Bernabé, B., y Franco, E. (2010). Estudio de la motivación del estudiante de Secundaria en Educación Física desde la perspectiva de Metas de Logro. Retrieved from http://oa.upm.es/7746/

Coterón, J., Franco, E., Pérez-Tejero, J., y Sampedro, J. (2013). Clima motivacional, competencia percibida, compromiso y ansiedad en Educación Física. Diferencias en función de la obligatoriedad de la enseñanza. Revista 
de Psicología del Deporte, 22(1). Retrieved from https://ddd.uab.cat/pub/revpsidep/revpsidep_a2013v22n1/ revpsidep_a2013v22n1p151.pdf

Durán-Aponte, E., y Elvira-Valdés, M. (2015). Patrones atribucionales y persistencia académica en estudiantes universitarios: validez de la Escala Atribucional de Motivación de Logro General (EAML-G). Revista Intercontinental de Psicología Y Educación, 17(2), 201-221. Retrieved from http://www.redalyc.org/ pdf/802/80247939011.pdf

Flores-Moreno, R., y Zamora-Salas, J. D. (2009). La educación física y el deporte como medios para adquirir y desarrollar valores en el nivel de primaria. Revista Educación, 33(1). https://doi.org/DO - 10.15517/ revedu.v33i1.536

Gómez, A., Gámez, S., y Martínez, I. (2011). Efectos del género y la etapa educativa del estudiante sobre la satisfacción y la desmotivación en Educación Física durante la educación obligatoria. Revista Ágora Para La Educación Física Y El Deporte, 13(2). Retrieved from http://uvadoc.uva.es/bitstream/10324/23716/1/AEFD-2011-2-efectosgenero-etapa.pdf

Gutiérrez, M. (2004). El valor del deporte en la educación integral del ser humano. Revista de Educación, 335. Retrieved from: http://www.revistaeducacion.mec.es/re335/re335_10.pdf

Halvari, H., Skjesol, K., y Bagøien, T. E. (2011). Motivational Climates, Achievement Goals, and Physical Education Outcomes: A Longitudinal Test of Achievement Goal Theory. Scandinavian Journal of Educational Research, 55(1), 79-104. https://doi.org/10.1080/00313831.2011.539855

Hellín, P., Moreno, J. A., y Rodríguez, P. (2006). Relación de la competencia motriz percibida con la práctica físicodeportiva. Revista de Psicología del Deporte, 15(2), 219-231. Retrieved from http://www.rpd-online.com/ article/view/232/232

Herrera, E., Morera, M., Araya, G., Fonseca, H., y Vargas, M. (2017). Análisis del estado físico y psicológico de la población escolar del cantón central de Heredia, Costa Rica. MHSALUD: Revista En Ciencias Del Movimiento Humano Y Salud, 13(2). https://doi.org/http://dx.doi.org/10.15359/mhs.13-27

Herrera, E., Delgado, L., Fonseca-Schmidt, H., y Vargas-Ramírez, P. (2012). Factores socio-afectivos relacionados con las dificultades escolares en niñas y niños "estrella" del programa psicomotricidad e intervención. MHSALUD: Revista En Ciencias Del Movimiento Humano Y Salud, 9(1). Retrieved from http://www.revistas.una.ac.cr/ index.php/mhsalud/article/view/3884/10988

Huertas, J. A. (1997). MOTIVACIÓN Querer aprender. Retrieved from http:// mateandoconlaciencia.zonalibre.org/TA_Huertas_Unidad_4.pdf

Lonsdale, C., Lester, A., Owen, K. B., White, R. L., Moyes, I., Peralta, L., y Lubans, D. R. (2015). An Internetsupported Physical Activity Intervention Delivered in Secondary Schools Located in Low Socio-economic Status Communities: Study Protocol for the Activity and Motivation in Physical Education (AMPED) Cluster Randomized Controlled Trial. BMC Public Health, 16(1), 17. https://doi.org/10.1186/s12889-015-2583-7

López, J. C., Álvarez, E. F., Pérez-Tejero, J., \& Molinuevo, J. S. (2013). Clima motivacional, competencia percibida, compromiso y ansiedad en Educación Física. Diferencias en función de la obligatoriedad de la enseñanza. [Motivational climate, perceived competence, commitment and anxiety in physical education. Differenc. Revista de Psicología del Deporte, 22(1), 151-157.

López, A., Camerino, O., y Castañer, M. (2015). Evaluar la motivación en la educación física, una aplicación con AMPET. Didáctica de La Educación Física, 47, 55-63. Retrieved from http://www.observesport.com/desktop/ images/docu/aj85rr9d.pdf

Márquez, M. (2015). Pertinencia del abordaje de los valores en la clase de educación física. Revista de Extensión Universidad En Diálogo, 5(2), 11-19. https://doi.org/http://dx.doi.org/10.15359/undre.5-2.1

Ministerio de Educación Pública (2013). Programas de Estudio de Educación Física, Primer y segundo ciclos de la Educación General Básica. San José, Costa Rica.

Moreno, J. A., González-Cutre, D., Martín-Albo, J., y Cervelló, E. (2010). Motivation and performance in physical education: An experimental test. Journal of Sports Science \& Medicine, 9(1), 79. Retrieved from http:// europepmc.org/articles/PMC3737955;jsessionid=1893F7220D8C391C66F2C0FE4187DFAA 
Naranjo-Pereira, M. (2009). Motivación: perspectivas teóricas y algunas consideraciones de su importancia en el ámbito educativo. Revista Educación, 33(2), 153-170. Retrieved from http://www.redalyc.org/ $\mathrm{html} / 440 / 44012058010 /$

Nishida, T., y Inomata, K. (1981). A Factor Analytical Study on Achievement Motives in Sports. Taiikugaku kenkyu (Japan Journal of Physical Education, Health and Sport Sciences), 26(2), 101-110. https://doi.org/10.5432/ jjpehss.KJ00003392765

Ntoumanis, N. (2001). A self-determination approach to the understanding of motivation in physical education. British Journal of Educational Psychology, 71(2), 225-242. https://doi.org/10.1348/000709901158497

Orozco, R. (2010). Motivación profesional en docentes de educación física de américa central. InterSedes, 11(20). Retrieved from https://revistas.ucr.ac.cr/index.php/intersedes/article/view/1014

Orozco, R. (2013). Motivación profesional, estilos de aprendizaje y satisfacción del estudiante en el contexto del aula de educación física. Universidad Estatal a Distancia. Retrieved from https://www.google.es/url?sa=t\&source=web\&rct=j\&url=http://repositorio.uned.ac.cr/ reuned/bitstream/120809/1005/1/Motivacion\%2520profesional\%2520estilos\%2520de \%2520aprendizaje.pdf\&ved=2ahUKEwim-82npMDaAhVFwlkKHa1eDE4QFjABegQIABAB\&usg=AOvVaw0TwxXJfK

Quevedo, V. J., Quevedo, R., y Bermúdez, M. (2009). Análisis de la motivación en la práctica de actividad físico-deportiva en adolescentes. Revista de Investigación En Educación, 6, 33-42. Retrieved from https:// dialnet.unirioja.es/servlet/articulo?codigo $=3216095$

Real Academia Española. (2018). Motivación. Retrieved from http://dle.rae.es/?id=Pw7w4I0

Romero, O. (1981). Motivación intrínseca, motivación de logro y valor incentivo de los estudios superiores. Retrieved from: http://www.saber.ula.ve/bitstream/handle/123456789/38180/ motivacion_intrinseca.pdf;jsessionid=D9B868560DD3F162DA2B48234EEA84F3? sequence $=1$

Rosales, F. R. (2011). La motivación, su estudio en la Educación Física. Lecturas: Educación Física y Deportes, 16(163). Retrieved from https://dialnet.unirioja.es/descarga/articulo/4103661.pdf

Ruiz-Juan, F., y Baena-Extremera, A. (2015). Predicción de las metas de logro en educación física a partir de la satisfacción, la motivación y las creencias de éxito en el deporte. Revista Iberoamericana de Psicología Del Ejercicio Y El Deporte, 10(2), 193-203. Retrieved from http://www.redalyc.org/html/3111/311137747004/

Ruiz, L. M., Graupera, J. L., Contreras, O. R., y Nishida, T. (2004). Motivación de logro en educación física escolar: un estudio comparativo entre cinco países. Revista de Educación, 333, 345-361. Retrieved from http:// www.revistaeducacion.educacion.es/re333/re333_16.pdf

Ruiz, L. M., Moreno, J. A., Ramón, I., y Alias-García, A. (2015). Motivación de Logro para Aprender en Educación Física: adaptación de la versión española del Test AM PET [ 1 ]. Revista Española de Pedagogía, (260), 157-176. Retrieved from http://content.ebscohost.com/ContentServer.asp? $\mathrm{T}=\mathrm{P} \& \mathrm{P}=\mathrm{AN} \& \mathrm{~K}=101375793 \& \mathrm{~S}=\mathrm{R} \& \mathrm{D}=$ ehh\&EbscoContent $=\mathrm{dGJyMNLe}$ 0SeprU40dvuOLCmr0\%2BeqLFSsqy4SbW

Ruiz, L. M. (2014). De qué hablamos cuando hablamos de Competencia Motriz. What do we talk about when we talk about Motor Competence? Acción Motriz, (2003), 37-44.

Sánchez Alcaraz, B., \& Gómez Mármol, A. (2016). Estudio de la motivación de logro y orientación motivacional en estudiantes de educación física. Educación Física y Deportes, 35-40. https://doi.org/10.5672/ apunts.2014-0983.es.(2016/2).124.04

Turabik, T., y Baskan, G. A. (2015). The Importance of Motivation Theories in Terms OfEducation Systems. Procedia - Social and Behavioral Sciences, 186, 1055-1063. https://doi.org/10.1016/j.sbspro.2015.04.006

Vargas-Pérez, P., y Orozco-Vargas, R. (2003). La importancia de la educación física en el currículo escolar. InterSedes, 4(7). Retrieved from https://revistas.ucr.ac.cr/index.php/intersedes/article/view/826

Wallhead, T. L., y Ntoumanis, N. (2004). Effects of a sport education intervention on students' motivational responses in physical education. Journal of Teaching in Physical Education, 23(1), 4-18. DOI: https://doi.org/10.1123/ jtpe.23.1.4 
Yamila, D., y Donolo, D. (2014). Factores clave en la promoción del compromiso en educación primaria. Un estudio sobre tasks, teachers and students. Congreso Iberoamericano de Ciencia, Tecnología, Innovación y Educación, 1-17. Retrieved from https://www.oei.es/historico/congreso2014/memoriactei/326.pdf

\section{BY-NC-ND}

